\title{
CONTRIBUTION A LA FAUNE HELMINTHOLOGIQUE SUD-AFRICAINE
}

Note préliminaire

\author{
Par Jean-G. BAER
}

Le matériel faisant l'objet de ce travail provient d'une grande collection de cestodes donnée à M. le professeur O. Fuhrmann par Sir Arnold Theiler, directeur du laboratoire de recherches vétérinaires de Prétoria. Dans cette note préliminaire nous ne nous occupperons que des espèces nouvelles pour la science et remettons à une publication ultérieure l'étude complète des cestodes de mammifères de cette collection provenant des régions voisines de Prétoria.

Tous les hôtes sont désignés d'après le catalogue des mammifères de Trouessart. Les types de cette collection seront déposés dans la collection de M. le professeur O. Fuhrmann.

\section{Lüheellidæ $\mathbf{n}$. fam.}

Lüheella pretoriensis n. g., n. sp.

\section{Hôte : Otocyon megalotis Desm.}

Il existe un seul exemplaire auquel il manque malheureusement la tête. La longueur est de $300 \mathrm{~mm}$., tous les segments étant plus larges que longs. La plus grande largeur mesurée est de $10 \mathrm{~mm}$.

La musculature longitudinale est très bien développée, de même la musculature transverse. Il y a des corpuscules calcaires en assez grand nombre dans tout le parenchyme.

Les deux nerfs longitudinaux sont très rapprochés l'un de l'autre, de sorte qu'ils se trouvent à $2 \mathrm{~mm} ., 7 \mathrm{du}$ bord dans les segments mesurant $9 \mathrm{~mm}$. de large. Le système excréteur présente un aspect tout à fait particulier, étant composé uniquement de dix vaisseaux longitudinaux, tous situés dans le parenchyme médullaire ; il ne se trouve qu'un seul vaisseau de chaque côté et en dedans du nerf.

Les testicules sont disposés sur une seule couche et occupent deux champs latéraux ne communiquant pas entre eux dans la par-

AnNales de Parasitologie, T. II, No 3 . - Juillet 1924, p. 239. 
tie antérieure du segment. Le canal déférent est très ondulé et débouche directement dans la poche du cirre. Cette dernière est assez musculeuse et présente dans sa partie distale une petite vésicule séminale. L'absence complète d'une vésicule séminale externe qui constitue le caractère fondamental de la famille des Diphyllobothriidæ Lühe, e. p., nous oblige à créer une nouvelle famille pour y rattacher ce nouveau genre.

Le vagin débouche postérieurement à la poche du cirre ; sa partie distale est renflée pour former un réceptacle séminal. L'ovaire se trouve sur la face ventrale dans la partie postérieure du segment. L'utérus forme une seule boucle de chaque côté de la ligne médiane, cette boucle se distend sous la pression des œufs en donnant l'aspect de deux sacs. Le pore utérin débouche en arrière du vagin, irrégulièrement à gauche ou à droite de la ligne médiane. La partie terminale de l'utérus est entourée de glandes utérines. Les glandes vitellogènes occupent la position habituelle. et sont interrompues sur les faces dorsale et ventrale au niveau des organes sexuels. Les œufs sont operculés et mesurent $61 \times 34 \mu$.

Diagnose de la familie des lebeel.lide n. Fam. - Pseudophyllides de taille moyenne; système musculaire bien développé ; pores génitaux au nombre de trois, situés l'un derrière l'autre sur la face ventrale ; vésicule séminale externe absente ; ovaire dans la partie postérieure du segment, formé de deux lobes réunis par un pont ; glande coquillière bien développée ; réceptacle séminal présent ; utérus en tube ondulé, dont la partie terminale est entourée de glandes ; œufs grands à coque épaisse, operculés.

Adultes chez les mammifères. Genre type Lüheella n. g.

La diagnose du genre est celle de la famille en tenant compte du système excréteur et de quelques autres détails. Adultes chez les carnivores. Espèce type Lüheella pretoriensis n. sp.

\section{Diphyllobothriidæ Lühe, 1899 , e. p.}

\section{Diphyllobothriinæ Lühe, 1899 , e. p.}

Diphyllobothrium Theileri n. sp.

Hôtes : Zibethailurus serval (Schreb.) ; Felis caffra Desm.

Ce cestode paraît assez fréquent d'après le nombre des exemplaires trouvés. La longueur varie entre 350 et $400 \mathrm{~mm}$. ; la plus grande largeur est de $3 \mathrm{~mm}$., 3. Le scolex de forme typique mesure $0 \mathrm{~mm}$., 53-0 mm., 86 de long, et $0 \mathrm{~mm}$., 25-0 mm., 3 de large. 
La musculature longitudinale est bien développée, tandis que la musculature dorso-ventrale ne l'est que faiblement. Les corpuscules calcaires se trouvent dans tout le parenchyme en assez petit nombre. Le système excréteur est composé de quatre vaisseaux longitudinaux situés dans le parenchyme médullaire et en dehors des nerfs longitudinaux. Il n'y a pas de vaisseaux périphériques, mais les quatre vaisseaux sont reliés entre eux par un réseau de vaisseaux secon. daires.

Les testicules sont très grands, en une seule couche et forment deux champs latéraux réunis entre eux par une seule rangée de testicules, dans la partie antérieure du segment. Le canal déférent est très ondulé, et débouche latéralement dans une vésicule séminale externe bien délimitée. La poche du cirre est assez grande et contieņt un gros cirre dont la cuticule est fendillée.

Le vagin débouche derrière la poche du cirre au fond d'un atrium assez profond; sa partie distale est renflée pour former un réceptacle séminal. L'ovaire est situé plutôt dorsalement. L'utérus est caractérisé par son grand diamètre et forme trois branches d'un côté et quatre de l'autre de la ligne médiane. Les glandes vitellogènes sont interrompues sur les faces dorsale et ventrale au niveau des organes sexuels. Les œufs ont une coquie épaisse et sont operculés, ils mesurent $57 \times 34 \mu$.

Cette espèce est voisine de D. Raillieti v. Rátz, (Kotlán, 1923).

\section{Anoplocephalidæ R. Blanchard, 1848}

\section{Anoplocephalinæ Fuhrmann, 1907}

Paranoplocephala acanthocirrosa n. sp.

Hôte : Otomys bisulcatus Cuv.

Plusieurs exemplaires de ce cestode furent recueillis chez un rat d'eau. La longueur varie de 45 à $60 \mathrm{~mm}$.; tous les segments sont plus larges que longs, la plus grande largeur étant de $5 \mathrm{~mm}$. La grandeur du scolex est assez variable suivant l'état de contraction.

La musculature longitudinale est bien développée et formée de deux couches concentriques; les musculatures transversales et dorso-ventrales sont également bien vísibles. Des corpuscules calcaires se trouvent dans tout le parenchyme. Les vaisseaux dorsaux du système excréteur sont situés en dehors des vaisseaux rentraux.

Les pores génitaux alternent irrégulièrement et les conduits génitaux passent dorsalement par rapport aux vaisseaux excréteurs 
et au nerf. La poche du cirre est grande et musculeuse, dépassant le vaisseau excréteur ventral. Elle contient une vésicule séminale bien différenciée; le cirre est robuste et toute sa surface est recouverte d'épines. Le canal déférent se dirige presqu'en ligne droite vers les testicules. Ces derniers forment une véritable grappe dans la moitié antiporale du segment. Il y a environ de 40 à 50 testicules dans chaque segment.

Le vagin débouche postérieurement à la poche du cirre et se renfle dans sa partie médiane en un assez grand réceptacle séminal. L'ovaire est situé du côté poral du segment et la glande vitellogène lui est postérieure. L'utérus est formé d'un tube transversal qui forme de 19 à 25 évaginations dans les directions antérieure et postérieure du segment. Les œufs sont pourvus d'un appareil piriforme bien développé.

\section{Fuhrmannella transvaalensis n. g., n. sp.}

\section{Hôte : Thryonomys swinderenianus Temm.}

Il manque malheureusement la tête au seul exemplaire que nous possédons. La longueur est d'environ $90 \mathrm{~mm}$. et la plus grande largevr de $7 \mathrm{~mm}$.

La musculature longitudinale est très fortement développée, disposée en deux couches; il en est de même des musculatures transversale et dorso-ventrale. De nombreux corpuscules calcaires se trouvent dans tout le parenchyme.

Les deux vaisseaux excréteurs dorsaux sont situés en dedans des vaisseaux ventraux. Les organes sexuels étant doubles, il y a deux pores génitaux qui débouchent au milieu du bord du segment. Les conduits génitaux passent dorsalement aux vaisseaux excréteurs et au nerf. Les testicules sont au nombre d'environ 200 et sont disposés sur deux à trois couches dorso-ventrales, remplissant tout le champ compris entre les ovaires et pouvant aussi dans certains cas se trouver en petit nombre du côté poral de ces derniers. Le canal déférent très ondulé est dilaté par le sperme et débouche dans une petite poche du cirre ; cette dernière n'atteint même pas le nerf longitudinal. Le cirre est inerme.

Le vagin débouche constamment postérieurement à la poche du cirre et des deux côtés du-segment. Dans sa partie distale, il se dilate pour former un réceptacle séminal. L'ovaire est en forme déventail, assez fortement lobé ; il est situé sur la face ventrale du segment; la glande vitellogène lui est postérieure et dorsale. L'utérus constitue le caractère principal de ce nouveau genre et 
présente une disposition qui n'a pas encore été observée chez les cestodes. Il est formé d'une série de cavités en forme de fer à cheval et qui sont disposées suivant le plan sagittal. Ces cavités sont reliées entre elles par un réseau d'anastomoses. De sorte que si l'on suppose un segment, vu en perspective, l'utérus apparaîtra comme un tunnel à l'intérieur duquel se trouvent les organes sexuels. A mesure que l'utérus se remplit-d'œufs, il se forme de nombreuses évaginations qui se frayent un passage entre les fibres des musculatures longitudinale et transversale et qui vont jusque sous la cuticule. A un stade plus avancé, des évaginations se forment vers l'intérieur du " tunnel ", de sorte que finalement tout le segment est bourré d'œufs. Ces derniers sont munis d'un appareil piriforme avec deux cornes.

Diagnose dU genRe Fuhruannella N. G. - Anoplocéphaliens à segments plus larges que longs; deux masses génitales par segment; conduits génitaux dorsaux par rapport aux vaisseaux excréteurs et aux nerfs; glandes interproglottidiennes absentes ; vagin postérieur à la poche du cirre de chaque côté du segment : testicules occupant tout le champ médian entre les ovaires, mais pouvant aussi se trouver en petit nombre du côté poral de ces derniers; utérus réticulé formant une sorte de "tunnel " antérodorso-ventral ; dans les segments âgés il se forme des évaginations passant entre les musculatures longitudinale et transversale, finalement tout le segment est bourré d'œufs ; ces derniers sont munis d'un appareil piriforme bien développé. Adultes chez les rongeurs. Type : Fuhrmannella transvaalensis n. sp.

\section{Linstowinæ Fuhrmann, 1907}

\section{Oochoristica ichneumontis n. sp.}

\section{Hôte : Herpestes gracilis Rüpp.}

Il n'existe que des fragments de deux exemplaires de ce ver, dont malheureusement aucun ne possède la tête. La plus grande largeur mesurée est de $4 \mathrm{~mm}$. Le système excréteur présente une particularité en ce que les vaisseaux ventraux sont très déplacés vers l'intéricur; les vaisseaux dorsaux sont situés en dehors des vaisseaux ventraux.

Les pores génitaux sont irrégulièrement alternes et les conduits génitaux passent dorsalement par rapport aux nerfs et aux vaisseaux excréteurs. Les testicules, au nombre de 90 à 100 environ, entourent l'ovaire sur trois côtés, ne se trouvant pas antérieu- 
rement à cet organe. Vu le grand déplacement des vaisseaux excréteurs, on trouve des testicules en dehors de ces derniers, sur le côté dorsal. Le canal déférent est très ondulé et débouche dans une poche du cirre qui ne dépasse guère le nerf longitudinal. Les capsules utérines ne contiennent qu'un seul embryon et sont disposées sur deux à trois couches. Elles remplissent le parenchyme médullaire et peuvent dans certains cas passer au delà des vaisseaux longitudinaux.

Vu la difficulté de distinguer le genre Oochoristica Lühe, 1898 du genre Linstowia Żschokke, 1899 , nous avons été amenés à ne maintenir dans ce dernier que, les deux espèces australiennes, à savoir : L. echidnæ (Thompson, 1893), Zschokke, 1899, et L. semoni (Zschokke, 1896). Ces deux espèces sont très bien caractérisées par le grand développement de la poche du cirre. Les quatre autres espèces de ce genre sont transférées dans le genre Oochoristica.

\section{Inermicapsifer aberratus n. sp.}

\section{Hôte : Mus moggi}

Il n'a été trouvé qu'un seul exemplaire de ce curieux ver et, vu son opacité, nous l'avons débité en coupes horizontales, de sorte que notre description ne pourra être complète. La longueur est d'environ $13 \mathrm{~mm}$. et la plus grande largeur de $0 \mathrm{~mm}$., 6. Ce qui caractérise cette espèce, c'est l'absence complète de testicules du côté poral du segment, tandis que du côté anti-poral se trouvent de 6 à 7 grands testicules de forme ovoïde. Le canal déférent s'en va en ligne droite rejoindre une assez petite poche du cirre. L'ovaire est situé franchement du côté poral. L'utérus est éphémère, comme c'est d'ailleurs le cas pour toutes les petites espèces de ce genre, et se résout en capsules contenant de 3 à 6 grands œufs. Il y a six de ces capsules par segment.

Nous avons profité d'un matériel abondant pour faire une revision complète de ce genre dont tous les types ont été obligeamment mis à notre disposition par leurs auteurs.

Nous avons cherché à établir la différence entre les deux genres Inermicapsijer Janicki, 1910 et Multicapsiferina Fuhrmann, $1921=$ Zschokkeella Ransom, 1909, ce dernier nom étant déjà occupé par une hydracarine. Nous avons trouvé 'qu'il existe la même différence qu'entre les deux sous-genres Paroniella Fuhrmann, 1920 et Ransomia Fuhrmann, 1920. C'est-à-dire que dans Inermicapsifer nous trouvons plusieurs œufs par capsule et dans Multicapsiferina un seul œuf par capsule. Vu l'identité de 
l'anatomie, nous avons réuni ces deux genres sous le nom de Inermicapsifer.

En faisant la revision des espèces, nous avons trouvé que le nombre de capsules par segment n'est pas un caractère spécifique ; il en est de même de la forme et de la taille des strobiles et des scolex. Les seuls caractères possédant une valeur spécifique sont la position du pore génital et la disposition des testicules. En appliquant ces conclusions aux nombreuses espèces décrites, nous avons pu distinguer douze espèces certaines, ce sont :

I. Linstowi (Parona, 1885). Syn. : Multicapsiferina Linstowi (Parona, 1885).

1. abeiratus $\mathrm{n}$. sp.

I. apospasmation Bischoff, 1912. Syn.: I. parvulus Bischoff, 1912.

I. arvicanthidis (Kofend, 1917). Syn. : Anoplocephala ? arvicanthịdis Kofend, 1917.

I. guineensis (Graham, 1908). Syn. : Zschokkeella guineensis (Graham, 1908), Southwell et Maplestone, 1921.

I. hyracis (Rudolphi, 1810). Syn. : I. criticus (Pagenstecher, 1877) ; I. capensis Beddard, 1912, et Hyracotænia hyracis Beddard, 1912.

I. interpositus Janicki, 1910. Syn.: I. abyssinicus Bischoff, 1912 ; 1. inter positus var. sinaitica Bischoff, 1912.

I. Norhalli Baer, 1924.

I. lopas Bischoff, 1912.

I. Pagenstecheri (Setti, 1897). Syn. : I. paronæ Bischoff, 1912 et Hyracotænia procaviæ Beddard, 1912.

I. prionodes Bischoff, 1912. Syn. : I. prionodes var, intermedia Bischoff, 1912.

I. Settii Janicki, 1910.

\section{Dilepinidæ Fuhrmann, 1907}

\section{Dipylidiinæ Stiles, 1896}

Dipylidium Fuhrmanni n. sp.

Hôtes : Zibethailurus serval (Schreb.) ; Felis caffra Desm.

Cette espèce présente un aspect curieux, tous les segments sauf les dix derniers étant plus larges que longs. La longueur totale est de $30 \mathrm{~mm}$. et la plus grande largeur de $2 \mathrm{~mm}$. Le scolex porte un rostellum armé de 14 à 16 rangées de crochets disposés en quinconce. Ces crochets sont de la forme typique des épines de rosier. 
Les corpuscules calcaires sont peu nombreux et semblent se concentrer dans les bords du parenchyme médullaire.

Les testicules sont grands et sont disposés sur deux, rarement sur trois couches. Au nombre de 40 à 50 , ils occupent la partie postérieure du segment et ne dépassent pas antérieurement les canaux déférents. Ceux-ci sont fortement enroulés sur eux-mêmes et mènent dans de grandes poches du cirre. Ces dernières sont situées obliquement dans le segment, la base de la poche étant dans la partie antérieure de ce dernier. Le cirre est mince et très long, plus long même que la poche qui le contient! Il en résulte qu'au repos et malgré les circonvolutions du canal à l'intérieur de la poche, le cirre lui-même est plissé en accordéon, afin de pouvoir être contenu tout entier dans la poche. Les capsules ovifères contiennent un seul œuf et ne dépassent jamais les vaisseaux excréteurs, se concentrant plutôt dans la région postérieure du segment.

Cette espèce est probablement identique à Dipylidium sp. Kofend, 1917.

\title{
Hymenolepinidæ Fuhrmann, 1907
}

Hymenolepis macroscelidarum n. sp.

Hôte : Macroscelides brachyrhynchus A. Smith

Longueur $34 \mathrm{~mm}$.; plus grande largeur $2 \mathrm{~mm}$. Le scolex porte un rostellum muni d'une seule couronne de 20 crochets longs de $19 \mu$.

Il y a deux testicules anti-poraux et un poral. Le canal déférent forme une assez grosse vésicule séminale avant de pénétrer dans la poche du cirre, puis en reforme une seconde à l'intérieur de cette dernière. Le cirre est inerme. L'atrium génital est pourvu à son orifice d'un puissant sphincter. Le vagin se dilate pour former un énorme réceptacle séminal. Les embryophores ont $36 \mu$ de diamètre.

\section{Tæniidæ Perrier, 1897 e. p.}

\author{
Tænia parva n. sp.
}

Hôte : Genetta ludia

Le plus grand exemplaire mesure $55 \mathrm{~mm}$. de long, la plus grande largeur étant de $3 \mathrm{~mm}$., 2 . Le scolex est muni d'un rostellum portant 
une double couronne de 44 crochets. Les grands crochets ont $361 \mu$ de long, avec une base de $228 \mu$ et les petits crochets $228 \mu$ de long, avec une base de $141 \mu$.

La musculature est extraordinairement bien développée et les corpuscules calcaires sont très nombreux dans tout le parenchyme. Il y a environ 500 testicules. L'utérus mûr présente de 7 à 12 branches latérales, lesquelles peuvent aussi se subdiviser. Les embryophores sont ovoïdes, et mesurent $27 \times 23 \mu$.

\section{Tænia hyænæ n. sp.}

Hôte : Hyæna brunea Thunb.

Longueur $300 \mathrm{~mm}$. ; largeur maxima $8 \mathrm{~mm}$. Le scolex porte un rostellum armé d'une double couronne de 32 à 38 crochets. Les grands crochets mesurent $223 \times 147 \mu$, et les petits $127 \times 83 \mu$.

La musculature est bien développée. Il ne se trouve que peu de corpuscules calcaires dans tout le parenchyme. Les testicules sont au nombre de 300 environ.

L'utérus présente de 12 à 14 branches latérales ; dans certains cas anormaux il y a dédoublement et l'on peut trouver de 20 à 24 branches latérales. Les embryophores sont ovoïdes et mesurent $34 \times 29 \mu$.

Laboraloire de Zoologie de l'Université de Nenchâtel (Suisse).

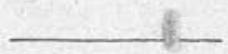

